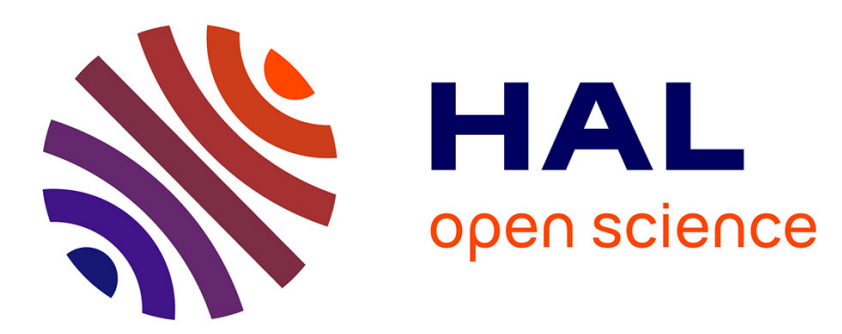

\title{
Histoire(s) et actualité du vodou à Paris. Hiérarchies sociales et relations de pouvoir dans un culte haïtien transnational.
}

\author{
Dimitri Béchacq
}

\section{- To cite this version:}

Dimitri Béchacq. Histoire(s) et actualité du vodou à Paris. Hiérarchies sociales et relations de pouvoir dans un culte haïtien transnational.. Studies in Religion/Sciences Religieuses, 2012, 41 (2), pp. 257 279. 10.1177/0008429812440973 . hal-01923110

\section{HAL Id: hal-01923110 https://hal.science/hal-01923110}

Submitted on 15 Nov 2018

HAL is a multi-disciplinary open access archive for the deposit and dissemination of scientific research documents, whether they are published or not. The documents may come from teaching and research institutions in France or abroad, or from public or private research centers.
L'archive ouverte pluridisciplinaire HAL, est destinée au dépôt et à la diffusion de documents scientifiques de niveau recherche, publiés ou non, émanant des établissements d'enseignement et de recherche français ou étrangers, des laboratoires publics ou privés. 


\section{Histoire(s) et}

actualité du vodou en

lle-de-France.

Hiérarchies sociales

et relations de

pouvoir dans un culte

haïtien transnational
Studies in Religion / Sciences Religieuses

$00(0) \mathrm{I}-23$

(C) The Author(s) / Le(s) auteur(s), 2012 Reprints and permission/

Reproduction et permission:

sagepub.co.uk/journalsPermissions.nav DOI: 10.1 I77/00084298I2440973

sr.sagepub.com

(\$SAGE

\section{Dimitri Béchacq \\ École des Hautes Études en Sciences Sociales}

Résumé : Cet article examine les conditions historiques, sociales et matérielles des pratiques du vodou à Paris et en Ile-de-France. Les enjeux de ces pratiques soulignent une dynamique de visibilité et d'invisibilité, d'inclusion et de fermeture qui détermine autant les relations humaines à l'intérieur du culte que les rapports avec une société française réfractaire au vodou. Sa présence à Paris remonte aux années 1960, avec les mises en scène inspirées du culte, et elle s'inscrit dans une histoire qui débute par les tournées des troupes folkloriques, formées à Port-au-Prince dans les années 1940. Les pratiques contemporaines du vodou en lle-de-France nécessitent des adaptations matérielles et rituelles. Elles relèvent autant d'une logique de protection que de relations de pouvoir fondées sur l'autorité religieuse et sur l'origine ethnique des pratiquants. Le vodou se révèle être un espace de compétition où les procédés de légitimation, véhiculés par des rumeurs, s'appuient sur des traditions et des territoires, sur la dimension économique et sur le respect des prescriptions rituelles. La circulation de ces rumeurs, de Paris à Brooklyn, suggère l'existence d'un espace transnational de moralité fondé sur des normes sociales et religieuses qui trouvent leur origine en Haïti.

Abstract: This article examines the historical, social and material conditions of the practice of Vodou in Paris and in the lle-de-France region. This practice can be

Corresponding author / Adresse de correspondance :

Dimitri Béchacq, L'École des hautes études en sciences sociales, 190-198 avenue de France, Paris

Email / Adresse électronique : d.bechacq@free.fr 
understood in terms of a dynamic of visibility and invisibility, of inclusion and exclusion which influences the human relationships within Vodou, as well as the resistance it has met in French society. Its presence in Paris goes back to the sixties, with theatre inspired by Vodou, and a history which begins with the tours of folk companies, trained in Portau-Prince in the forties. The contemporary practice of Vodou in the lle-de-France has involved material and ritual adaptations. It is founded as much upon a logic of protections as upon power relationships between religious authorities and the ethnic origins of Vodou practitioners. Vodou is revealed to be a competitive space where the process of legitimization, aided by rumours, is based on traditions and territories, on the economic dimension and on respect for ritual instruction. The circulation of these rumours, from Paris to Brooklyn, suggests the existence of a transnational space of morality based on social and religious norms which have their origin in Haiti.

\section{Mots clés}

Vodou, Haïti, lle-de-France, Brooklyn, migration, spectacles, adaptations rituelles, rumeurs, relations de pouvoir

\section{Keywords}

Vodou, Haiti, Ile-de-France, Brooklyn, migration, shows, ritual adaptations, rumours, power relations

\section{Introduction}

« C'est dix fois plus que ce que les gens imaginent le vodou. En même temps, je me dis : heureusement qu'ils ont cette image négative du vodou, parce que si les gens savaient réellement l'intérêt qu'il y avait à être et à pratiquer le vodou, ça finirait comme le bouddhisme, comme la religion catholique, ça finirait par devenir purement commercial [...]. Les gens qui sont ici sont triés sur le volet. Depuis que je suis toute petite, ma mère doit avoir initié peut-être 250 personnes en France. » (Manbo R., 12 nov. 2003)

R. est une manbo d'une trentaine d'années qui est arrivée en France à l'âge de quatre ans avec ses deux frères pour rejoindre son père et sa mère, manbo $\mathrm{M}$. Cette dernière est à la tête de deux temples. L'un se situe dans une ville à 30 kilomètres de Port-au-Prince, l'autre est en banlieue parisienne, dans la cave d'un pavillon où sa famille spirituelle, composée principalement de Guadeloupéens et de Martiniquais, se réunit régulièrement pour pratiquer le vodou. Cet extrait de témoignage souligne l'influence de la réputation sulfureuse du culte et de ses enjeux économiques sur la nécessité d'opérer une sélection parmi les candidats que sa mère a initiés en Ile-de-France. ${ }^{1}$ La présence de cette religion dans cette région est liée à la migration haïtienne qui a pris plusieurs formes. La plus connue est celle d'une migration d'origine populaire qui s'est accentuée à partir de la fin des années 1970; la moins connue, parce que plus confidentielle et moins documentée, est liée aux tournées mondiales des troupes folkloriques qui ont débuté dans les années 1940.

Entre ces deux formes d'un même phénomène, les pratiques transnationales du vodou donnent à voir la diversité de ses usages sociaux et politiques. Les adaptations du culte 
destinées à le rendre accessible à un public non haïtien sont animées par une dynamique de visibilité et d'invisibilité, par un processus d'inclusion et d'exclusion qui délimitent les contours d'une appartenance haïtienne fondée sur cette religion. Loin de n'être qu'un appendice naturel des classes populaires haïtiennes pour lesquelles l'Afrique serait la seule planche de salut imaginaire et la seule grille de lecture savante (Apter, 2002; Heusch, 1989; Hurbon 1979 : 124; Hurbon, 1987 : 149; Métraux, 1995 [1958] : 11), l'histoire et l'actualité du vodou en Ile-de-France montrent au contraire la prégnance des luttes de pouvoir et de prestige dans l'articulation étroite entre cette religion et l'identité haïtienne. En effet, les mises en scène du vodou à Paris à partir de la fin des années 1960, la présence contemporaine d'Antillais dans le culte, et la circulation des rumeurs qui forme un espace transnational de moralité, témoignent des hiérarchies sociales et des relations de pouvoir qui structurent le vodou haïtien d'un bord à l'autre de l'Atlantique.

\section{Des troupes folkloriques aux filières migratoires : usages politiques et sociaux des mises en scène du vodou haïtien}

Le vodou est au cœur des relations entre des cultures de classe car il fut construit, sous une forme folklorique et par certains des membres de l'élite haïtienne, comme un produit culturel exportable à l'étranger. Leur investissement dans le culte montre qu'il est un espace de pouvoir pouvant servir au développement d'une politique culturelle nationale offensive et à des ambitions personnelles. Pour comprendre par quels chemins le vodou est arrivé en France à la fin des années 1960, il faut remonter brièvement à la période allant des années 1920 aux années 1950 .

Pendant ces décennies, Haïti fut un véritable carrefour d'acteurs impliqués dans la valorisation de la culture populaire, telle qu'elle avait été prônée par des intellectuels haïtiens à partir des années 1920, dont Jean Price-Mars (Hoffmann, 1990 : 185 ; Price-Mars, 2009 [1928] ; Tippenhauer, 2008 : 100). Certains d'entre eux avaient été formés dans les capitales occidentales où les productions artistiques inspirées de l'Afrique connaissaient un franc succès (Capone, 2006 : 85-91; Fabre, 1977 ; Gaillard, 1993 : 13 ; Nicholls, 1996 [1979] : 158). Pendant cette période fondatrice pour l'ethnologie haïtienne et l'anthropologie culturaliste nord-américaine, ces disciplines appréhendèrent le vodou à travers un double processus : l'inclusion du culte dans l'ordre de la civilisation et son exclusion, du moins son confinement, en tant qu'altérité culturelle alors identifiée comme relevant du folklore. Il en est ressorti la formation d'un vodou savant, dans laquelle l'influence des contextes d'observation et d'énonciation, soumis aux rapports de pouvoir marquant le paysage social et politique haïtien, était minorée (Béchacq, 2006 et 2008). La prégnance du paradigme africaniste dans l'analyse des productions culturelles haïtiennes s'appuya sur ce dénominateur commun permettant de rattacher Haïti à l'Afrique, la race noire, élément central dans le triptyque examiné par Gérarde Magloire et Kevin Yelvington (2005) : « Afrique-race-vodou ».

La construction de ce vodou savant émergea au sein d'un réseau de chercheurs, d'artistes et de serviteurs du culte dont certains furent mobilisés dans le mouvement folklorique haïtien, endossant parfois la double casquette d'informateurs pour les ethnologues et de professeurs de danse pour les étudiants de l'Institut d'ethnologie créé au début des années 1940 (Ramsey, 2005 : 176). Karen Richman a souligné comment la 
présence de chercheurs haïtiens et étrangers dans les services vodou pouvait orienter leur déroulement, renforçant ainsi « la tendance vers la codification de performance rituelle complexe » (Richman 2005 : 123). Kate Ramsey (2005) a montré quant à elle comment la prohibition du vodou par les législateurs, dans les années 1930 et 1940, a encouragé la floraison de travaux ethnographiques et de spectacles folkloriques. L'épicentre de cette dynamique fut le Bureau d'ethnologie, créé en 1941, et sa revue, le Bulletin du Bureau d'ethnologie (Charlier-Doucet, 2005). Les spectacles folkloriques mettaient en scène des chorégraphies et des chants inspirés du vodou qui étaient interprétés selon des normes esthétiques issues de la musique classique, dans la lignée de l'éducation «à l'occidentale » de l'élite (Largey, 2006). Ces spectacles étaient forts appréciés des touristes et de la bourgeoisie haïtienne dont certains des membres étaient à l'origine de la création de nombreuses troupes (Oriol, Viaud et Aubourg, 1952 ; Smith, 2009 : 59). La plus connue d'entre elles fut la Troupe Folklorique Nationale créée à l'occasion de l'Exposition internationale et du Bicentenaire de Port-au-Prince, en 1949-1950.

Ces événements, dont la publicité sur le continent américain fut assurée par le Bureau du Tourisme créé en 1947 (Smith, 2009 : 107), attirèrent de nombreux touristes et promoteurs culturels étrangers qui invitèrent les troupes haïtiennes à se produire en dehors d'Haïti. Ainsi, pour les gouvernants haïtiens, le Bicentenaire et l'Exposition internationale furent une vitrine des compétences artistiques haïtiennes, véritables cartes de visite pour assurer la promotion d'Haïti à l'étranger, notamment aux États-Unis. La Troupe Folklorique Nationale, dirigée alors par Jean Léon Destiné, fut invitée en 1951 au Festival des Cerisiers en Fleurs, connu sous le nom de National Folk Festival, à Washington. ${ }^{2}$ La même année, celle qui devint le personnage central du célèbre ouvrage de Karen McCarthy Brown, Mama Lola, fut recrutée, à l'âge de 16 ans, comme chanteuse au sein de la troupe officielle (Brown, 2001 [1991] : 237). Mme Blanchet s'était déjà rendue en 1941 dans la capitale américaine avec sa troupe privée Legba Singers pour se produire à la Pan American Conference (Largey, 2006 : 203). Elle y retourna en 1951, à la tête d'une autre troupe, Haïti Chante, qui se produisit ensuite dans les hôtels et les théâtres de Port-au-Prince (Yarborough, 1959: 5).

Dans les années 1950, les nombreuses troupes folkloriques créées lors de la décennie précédente, devinrent le bras armé de la politique nationale en matière de promotion culturelle à l'étranger. Cela permettait de vanter les atouts d'Haïti sur le mode de «l'authenticité », au même titre que les arts dits « primitifs » inspirés du vodou (Richman, 2008), dans un secteur en pleine expansion, le tourisme, au sein duquel les pays de la Caraïbe étaient en compétition (Smith, 2009: 107 et 164). L'on peut donc véritablement parler d'une esthétique politique du vodou, dans la mesure où l'image qui en était donnée servait des intérêts politiques. Cependant, la matière première de ces divertissements fut puisée dans la société rurale, mais les paysans ne furent pas les destinataires des retombées économiques générées par cette esthétique politique du vodou. En outre, ces troupes étaient dirigées par des membres de l'élite haïtienne et les artistes qui mettaient en scène des chants et des danses inspirées du vodou étaient élevés au rang d'ambassadeurs de la culture haitienne. ${ }^{3}$ Cette tâche ne pouvait revenir à des paysans ou à des oungan et des manbo d'origine sociale modeste, comme le révèlent les propos de la directrice d'une école de danse dont certains des élèves se produisirent à l'étranger : 
«En réalité, nous n'avions pas de troupes rurales qui sont parties à l'étranger. C'étaient des gens, si vous voulez, de la classe moyenne, qui étaient là et qui aimaient ça. [...] On a l'air de croire que la paysannerie, elle peut danser, mais elle ne peut pas parler, elle ne peut pas être ambassadeur et quand on voyage, on est obligé d'être ambassadeur car quand on vous pose des questions et qu'on répond : "m'pa kone" [je ne sais pas], ça n'a pas de sens mon cher ». (Port-au-Prince, 2 février 2007)

Les mises en scène du folklore s'inscrivaient donc dans les hiérarchies sociales haïtiennes. Le développement du mouvement folklorique fut encouragé sous la présidence de Dumarsais Estimé (1946-1950), chef de file des « Authentiques », qui fut marquée par l'arrivée des noirs dans les institutions publiques. Sydney Mintz et Michel-Rolph Trouillot soulignent que cette récupération du culte par l'élite révèle la pérennité des relations de pouvoir qui s'exprimaient ici dans les usages du vodou à des fins de propagande nationale :

Plutôt, il s'agit de mettre l'accent sur le fait que la relation entre les élites haïtiennes et le vodou a toujours été marquée par l'expropriation. Vue sous cet angle, la nouvelle approche menée par Estimé et rétablie aujourd'hui par ses avatars actuels correspond à un modèle centenaire d'usage condescendant. (Mintz et Trouillot, 1998 [1995] : 143)

Ce rapport pour le moins ambigu entre l'élite et le vodou, appréhendé comme le symbole de la « culture populaire », relèverait d'une tendance plus générale marquant cette classe sociale gouvernée, selon Louis Naud Pierre, par le « chaos des prétentions » où « l'élite socioculturelle transforme le patrimoine culturel en fond de commerce exotique » (Pierre, 2008 : 60). Les troupes folkloriques haïtiennes voyageaient à l'étranger et elles se produisaient dans les nombreux hôtels pour offrir aux touristes, surtout nord-américains, des vodoo shows qui renforçaient leurs stéréotypes à l'égard du culte (Goldberg, 1981). ${ }^{4}$

Ces tournées étaient sous-tendues par des enjeux économiques et migratoires pour les artistes et serviteurs du vodou qui y participaient. Elles étaient un moyen pour récolter l'argent nécessaire à leur installation en Haïti ou pour quitter le pays. Ce fut le cas de Cicéron Saint-Aude, oungan et fils adoptif de la manbo Lorgina Delorge (BeauvoirDominique, 2005 : 1) dans le temple de laquelle Alfred Métraux réalisa son enquête. Ce dernier précise qu' « un des meilleurs acteurs de la troupe de Katherine Dunham ${ }^{5}$ était précisément un jeune houngan qui venait de "prendre l'asson" et espérait, en parcourant le monde, mettre de côté la somme nécessaire à son installation » (Métraux, 1995 [1958] : 63). Ce jeune oungan, Cicéron Saint-Aude, se produisit avec Katherine Dunham au Théâtre des Champs-Élysées à Paris en 1951. ${ }^{6}$ À l'issue de l'une des représentations de leur spectacle, intitulé Southland (Capone, 2006 : 93), il fut sollicité par Alfred Métraux pour diriger une cérémonie privée dans un atelier parisien (Métraux, 1978 : 345). Les tournées des troupes folkloriques furent également un moyen pour quitter Haïti, alors que sévissait la dictature des Duvalier (1957-1986). Loïs Wilcken, manager de la Troupe Makandal, analyse les effets de la dictature sur la formation de troupes dans la ville de New York et elle souligne que dans les années 1970-1980, « la migration vers les États-Unis emporta un nombre substantiels d'artistes folkloriques et ils créèrent rapidement des compagnies à New York » (Wilcken, 1998 : 170). Le témoignage de Fritz 
Jolicœur, directeur de la Troupe Folklorique Nationale de 1977 à 1984, montre que la troupe officielle était composée d'artistes des compagnies privées, car les tournées à l'étranger étaient une opportunité pour rejoindre la famille résidant à l'étranger :

«Les danseurs qui formaient la Troupe Nationale venaient en grande partie de la troupe de Viviane Gauthier. À un certain moment, je suis allé aux États-Unis, je crois que c'était en 1980. Je suis parti avec vingt-cinq personnes et neuf seulement sont revenues en Haïti ». (Port-au-Prince, 22 janvier 2007)

Qu'en était-il des tournées des troupes folkloriques en Europe et plus particulièrement en France?

Viviane Gauthier devint professeur de la Troupe Folklorique Nationale en 1971 et elle se rendit en France cette année-là, mais dans le cadre de la tournée européenne de la troupe Bakoulou, créée en 1959 par Odette Wiener Latour. En 1959, Félix MorisseauLeroy et sa Compagnie Nationale d'Art Populaire Bacoulou, furent invités à Paris sur la scène du Théâtre des Nations (Peslin-Ursu, 2009 : 210). Claude Planson, qui fut directeur artistique de ce théâtre de 1957 à 1964, y rencontra Mathilda Beauvoir. Cette dernière a un parcours singulier. Née à la fin des années 1930 en Haïti, arrière-petitefille du président Florvil Hyppolite (1889-1896), fille de médecin, elle est également la demi-sœur de Max Beauvoir, l'Ati Nasyonal ou Guide Suprême du vodou haïtien intronisé en janvier 2008. Elle fut formée chez les sœurs de Sainte-Rose de Lima, l'une des institutions catholiques de Port-au-Prince les plus prisées par les grandes familles bourgeoises haïtiennes (Planson, 1975 [1974] : 267), et à Paris, elle suivit les cours de danse de Maurice Béjart. Roger Bastide souligna la filiation au niveau des techniques chorégraphiques entre Katherine Dunham et Mathilda Beauvoir (Bastide, Morin et Raveau, 1974 : 56-57), qui dirigeait des spectacles dans le club Le Vaudou, situé à Pigalle et tenu par son époux, Claude Planson. Ce dernier le qualifie de «premier "hounfor" [temple] européen » (Planson, 1975 [1974] : 148). Dans le cas des mises en scène folkloriques inspirées du vodou, on peut se demander si nous avons toujours affaire à une religion. Dans ce club, la frontière entre le folklore et le vodou était encore plus ténue, car il s'agissait de rituels religieux se déroulant sur une scène, programme et transe de possession à l'appui, pendant le dîner des spectateurs. ${ }^{7}$ De plus, Mathilda Beauvoir recrutait en Haït, notamment chez Katherine Dunham, des danseurs et des danseuses qu'elle conduisait en France pour se produire dans le club Le Vaudou. Au début des années 1970, manbo F. arriva à Paris de cette façon. Elle devint manbo en 1984 :

« Je dansais le folklore en Haïti et j'ai fait un peu de ballet. Je suis arrivée en France, on était plusieurs artistes. Je suis rentrée ici avec Mme Claude Planson, Mathilda Beauvoir. On était six danseuses et deux danseurs. On a dansé deux années dans le club et après on a dansé à l'Olympia. J'ai arrêté deux ans après, de temps en temps je faisais des chorégraphies et la dernière fois c'était en 1984. [ . . . ] J'ai arrêté, mais des fois l'Ambassade d'Haïti m'appelait s'il y avait un contrat, c'était mon métier, donc j'ai accepté. Mais si je n'avais pas envie, je ne mélangeais pas [...]. Artiste, c'est un métier, mais j'ai laissé tomber, je suis manbo maintenant, mais je ne mélange pas parce que je ne veux pas ... si quelqu'un me voit sur scène et qu'après il vient chez moi, il peut penser que je joue la comédie. C'est pour ça que 
je me suis arrêtée parce que être sur scène et être avec une manbo, ce sont deux choses différentes ». (22 mai 2002)

Manbo F. craignait la confusion entre le genre artistique et le genre religieux, et des effets de celle-ci sur sa crédibilité auprès de potentiels clients. Les expériences artistiques et religieuses de Mathilda Beauvoir et de manbo F. posent la question de la frontière entre visibilité et invisibilité : il est des choses à ne pas dire, à ne pas faire et surtout, à ne pas montrer. De plus, dans l'ouvrage que Claude Planson consacra à la description de son parcours initiatique auprès de son épouse, il décrit le rythme soutenu de leurs voyages entre Haïti et la France dont l'objectif était de recruter des initiés destinés à se rendre avec eux à Paris. ${ }^{8}$ L'on peut véritablement parler de filières migratoires organisées à partir d'une pratique religieuse et artistique car plusieurs dizaines de personnes sont ainsi parties d'Haïti. Cependant, certaines d'entre elles auraient servi comme domestiques, dans des conditions proches de l'esclavage moderne, pratique condamnée par la loi française. Ces dérives ont fait l'objet d'un reportage télévisé sur la chaîne TF1, diffusé le 3 novembre 2002 et il fut relayé par un journal haïtien à Paris sous le titre « Esclavage moderne en France. Une Haïtienne exploitée pendant 20 ans ». ${ }^{9}$ En outre, le témoignage de Claude Planson montre à quel point ces serviteurs du vodou étaient réduits à l'état d'objet, d'individus dénués de libre arbitre quant à la pratique même de leur religion. ${ }^{10}$ Le parcours de manbo F. illustre tout le contraire : elle exerce aujourd'hui la profession d'aide soignante dans un hôpital, elle reçoit des clients pour des leson [consultation] dans son appartement situé dans une ville de la Seine-Saint-Denis et elle se rend régulièrement en Haïti pour les cérémonies qu'elle ne peut réaliser en France. Si l'histoire qui a mis en cause Mathilda Beauvoir a jeté un voile trouble sur sa réputation et a accentué les critiques d'Haïtiens quant à ses mises en scène spectaculaires du vodou, elle éclaire cependant l'un des chemins par lesquels le culte est arrivé en France.

\section{Les pratiques du vodou haïtien en Ile-de-France : des adaptations rituelles au maintien discret de frontières ethniques}

Observer les pratiques du vodou haïtien à Paris en étant blanc, Français et non initié a constitué un véritable défi dans l'enquête ethnographique. J'ai préféré décliner les invitations à être initié car j'avais l'intime conviction que l'initiation appelait à être au cœur de relations de pouvoir. ${ }^{11}$ La distance nécessaire pour conserver ma liberté d'écriture m'empêcha cependant d'accéder à certaines données, autant rituelles que relationnelles, les initiés étant liés par une intimité et une solidarité qui sont similaires à ceux d'une famille. Le vodou est en effet une religion initiatique dont le pivot est le secret. En outre, la question de la sorcellerie est au cœur de la réputation sulfureuse du culte. ${ }^{12}$ Les serviteurs du vodou cachent souvent leur appartenance religieuse, de peur d'être vus par des étrangers comme des sorciers en puissance. Cependant, l'ambivalence est au cœur du pouvoir des oungan et des manbo, capables de faire le bien comme le mal, ce qui est désigné par l'expression « travailler des deux mains ». De plus, la sorcellerie assure au culte autant une publicité négative, avec les nombreux stéréotypes produits à son encontre, que positive : réputé être puissant, le vodou attire de nombreux clients. Ces deux dimensions sont de fait indissociables. Si l'invisibilité est un gage de protection 
contre le regard extérieur, la visibilité peut être pensée dans une optique de gain, qu'il soit politique, personnel ou financier. Dès lors, la notion de secret et cette question de la sorcellerie sont traversées par un subtil équilibre entre accessibilité et fermeture, visibilité et invisibilité.

La présence du vodou en Ile-de-France nécessite des adaptations matérielles et rituelles au contexte urbain, lequel implique une triple discrétion. Une discrétion qui entoure les échanges économiques qui, le plus souvent, circulent de façon informelle; une discrétion imposée à cette religion chantée et dansée avec le respect de la vie en collectivité; enfin, une discrétion qui peut affecter les rituels avec l'interdiction des sacrifices par la loi française.

Le vodou constitue à proprement parler un espace économique qui aurait émergé dans des conditions sociohistoriques particulières. À partir du milieu du 20e siècle, le démantèlement des parcelles familiales sous l'effet de la pression démographique et l'exode rural a favorisé le rôle des oungan et des manbo comme intermédiaires avec les esprits (Béchacq, 2004 : 42-43). À la suite des travaux de Gérald Murray, Karen Richman va plus loin en montrant que l'expression «pran ason » ["prendre l'asson»], qui désigne dans le sud d'Haïti la fin du cycle d'initiation et l'accession au grade de manbo et de oungan asogwe, révélerait la tendance à la commercialisation des pratiques du vodou à partir de cette même période. Devenir oungan ou manbo peut donc être le fruit d'une démarche personnelle, parfois doublée d'une visée utilitariste (Richman, $2005: 116-124) .{ }^{13}$ De plus, l'attrait de touristes pour le vodou et le développement d'un tourisme religieux en Haïti dont Max Beauvoir fut l'un des acteurs (Cosentino, 1993, 1998 [1995] : 43 ; voir également Richman, 2008) - interrogent l'influence du contexte économique haïtien sur la commercialisation des pratiques religieuses avec l'émergence d'un marché de l'initiation.

De l'avis de plusieurs oungan et manbo rencontrés à Port-au-Prince et à Paris, les consultations de voyance [leson] représentent une rentrée plus ou moins régulière d'argent et constituent leur fond de commerce. Oungan R. a vécu en France de 1992 à 2001 et il vit aujourd'hui aux États-Unis, tout en voyageant régulièrement en Haïti et en Europe où résident certains de ses initiés. Il assume pleinement le fait de tirer des revenus de ses compétences religieuses, dès lors que le service des esprits exige des dépenses importantes :

« On a besoin de faire de l'argent avec le vodou, il ne faut pas être hypocrite. C'est notre façon de vivre, c'est notre gagne-pain, des fois les mystères ne te laissent pas faire autre chose que du vodou, que des consultations, que des travaux mystiques, mais il faut bien vivre, il faut bien payer les factures, il faut bien manger [... ]. Le vodou, les mystères ont besoin d'un beau temple, ils ont besoin de beaux foulards, ils ont besoin de bonnes liqueurs, ils adorent les belles bougies, les parfums. Pour moi, servir les mystères, c'est un luxe, il faut avoir de l'argent pour servir les lwa». (Brooklyn, 19 novembre 2004)

Le temple dans lequel j'ai enquêté de 2002 à 2007 est situé dans la cave d'un pavillon de la banlieue parisienne. Il est tenu par manbo M., arrivée en France au début des années 1970, et par ses trois enfants, une fille et deux garçons, qui sont manbo et oungan. À la différence d'un grand nombre de leurs pairs, ils parviennent à vivre des revenus tirés des pratiques du vodou. Connue des services de police et des impôts, manbo M. est taxée sur 
ses revenus, car elle est déclarée en tant que profession libérale, ce qui lui permet de déduire de son imposition les achats de produits pour la réalisation des cérémonies. Cela conduit également des inspecteurs des impôts à venir y assister pour vérifier que les revenus déclarés correspondent bien au nombre de participants. L'un des services organisés en l'honneur des Gédé dans le temple de manbo M. coûta $1500 €$ : aux deux boucs noirs qui furent sacrifiés, s'ajoutèrent les sodas et les bouteilles d'alcool, servis à profusion. Le lendemain, les animaux sacrifiés furent cuisinés et servis aux membres de la famille spirituelle et aux personnes extérieures au temple. Comme l'a souligné Karen McCarthy Brown dans son analyse de l'économie rituelle du vodou, ces repas attestent des liens de réciprocité entre les personnes (Brown, 1998 [1995] : 209-210). À ces coûts, il faut également ajouter, comme dans le cas d'un service en l'honneur des Gédé dirigé par le ougan R. à Brooklyn, le paiement des prestations des trois tambourineurs, ce qui lui coûta 1200 \$ pour les deux soirs du service. Devant de telles dépenses, il n'est pas rare que les pitit fey [initiés] du oungan ou de la manbo participent aux frais de la cérémonie.

Le vodou est un espace économique, car il engage différents protagonistes dans une configuration au centre desquelles on retrouve la relation entre les oungan, les manbo et leurs esprits, relation de type don/contre-don d'où ils tirent leur légitimité à réaliser des profits avec le culte. L'ensemble des relations sociales et des liens d'interdépendance qui se déploient dans le vodou déterminent l'intensité des échanges économiques dont la pérennité repose sur une dynamique de circulation d'argent, de biens matériels et de prestations religieuses. Cette dynamique est transnationale quand le oungan ou la manbo possède un temple à l'étranger et un autre en Haïti, à l'instar de manbo M. La solidarité qui lie les membres d'une même famille spirituelle fait également partie des devoirs du migrant, d'autant plus quand celui-ci rentre en Haïti. La fille de manbo M. raconte comment sa mère aide les gens de sa localité :

«Elle fait beaucoup de choses là-bas, notamment de l'eau dans le quartier, de l'électricité, de la nourriture, elle aide des gens dans le commerce. Une fois, on lui a demandé d'être député dans le canton. Mais ma mère ne fait pas de politique, elle a dit: "si vous voulez que je vous aide, je peux vous aider mais je ne fais pas de politique” », (Paris, 15 mars 2003)

Comme l'avait remarqué Alfred Métraux (1995 [1958] : 55) à son époque, devenir oungan ou manbo assure une position sociale en vue dans la communauté locale, ce qui est d'autant plus vrai pour le migrant qui peut vivre des revenus tirés de ses activités religieuses.

En Ile-de-France, rares sont les vodouïsants qui possèdent suffisamment d'espace dans leur habitat pour réaliser des services en bonne et due forme, avec tambours et sacrifices. Dès lors, plusieurs adaptations sont possibles. Le service des esprits peut, en Haïti comme à l'étranger, se réaliser avec des moyens élémentaires : quelques bougies, une offrande de rhum après que la bouteille eut été présentée aux quatre coins cardinaux, puis des prières catholiques et des demandes faites au $l w a$. La faiblesse des revenus d'une majorité de migrants haïtiens, qui en envoient une partie à leur famille résidant en Haïti, et l'offre immobilière en Ile-de-France, dominée par des prix élevés, font que de nombreux serviteurs vivent dans des appartements de faible superficie. Dans ce contexte, la réalisation de sacrifices est problématique. Oungan R., qui habitait dans un petit studio 
parisien, détaille les précautions qu'il devait prendre, notamment par rapport à la façon dont des étrangers pourraient juger les serviteurs à cause des sacrifices :

«Quand tu arrives dans un pays comme la France et que tu es vodouïsant, automatiquement tu vas adapter. [...] Tu habites en appartement, tu ne peux pas faire de bruit, aller contre l'ordre établi. [... ] Il y a des lois qui interdisent de sacrifier des animaux dans les appartements et bien .... tu fais attention. Ça arrive qu'on en fasse, mais tu fais attention, ce sont des cérémonies secrètes, ce sont des cérémonies propitiatoires, c'est entre initiés seulement. Les étrangers arrivent plus tard [...]. Ce n'est même pas le sacrifice en lui-même que les gens voient, c'est tout ce qu'ils vont dire, vont comprendre et interpréter. Je crois que beaucoup de vodouïsants ont très peur de ça ». (Paris, 7 janvier 2002)

Quant à manbo F., elle ne réalise dans son appartement que les rituels ne nécessitant pas de sacrifices, tels que les mariages mystiques et les actions de grâce. Pour réaliser des services tels que les manje lwa, qui impliquent de réunir du monde et de sacrifier plusieurs animaux, elle retourne en Haïti, ce qui suppose d'avoir des ressources financières suffisantes et de posséder des documents administratifs en règle, ou la nationalité française, pour voyager librement. Si, en Haïti, les services peuvent se dérouler sur plusieurs jours, en France, cela est incompatible avec les obligations professionnelles de la plupart des migrants. Mais il existe une prière qui condense les salutations aux différents lwa et permet de raccourcir la durée des services qui, le plus souvent, ont lieu le week-end :

«On ne salue pas tous les lwa mais il y a une façon pour pouvoir saluer beaucoup de lwa ensemble [...]. On est obligé de passer par Legba, obligatoirement, il faut qu'on ouvre les portes, il faut qu'on salue les enfants, les jumeaux [marassa], mais pour les autres, on condense. On prend dix à quinze lwa et on les salue tous ensemble. Ce n'est pas vraiment une adaptation qu'on a créée nous-mêmes, on l'appelle le minokan, c'est l'union des lwa, on salue les principaux $l w a$ et les autres, on les met en minokan ». (Oungan R., Paris, 7 janvier 2002)

Avec le minokan et le regleman, liturgie spécifique à chaque initié lui permettant d'insérer des éléments personnels dans le rituel dont les salutations à ses propres lwa, le vodou est structuré de telle sorte qu'il permet les adaptations aux contraintes d'un nouvel environnement et de nouvelles temporalités. Si les éléments nécessaires aux rituels bougies, encens, parfums, chromolithographies, etc. - sont disponibles dans certaines boutiques du quartier de Belleville à Paris, les fey [feuilles], écorces et racines - ingrédients essentiels autant dans les rituels que dans les traitements thérapeutiques - n'existent pas en France. Ainsi, les vodouïsants d'Ile-de-France sont en contact régulier avec leurs pairs de Miami, cette ville jouant un rôle central dans les réseaux des vodouïsants, autant du fait de son climat tropical que de l'importance numérique des migrants haïtiens. Au-delà de l'adaptation des rituels aux contraintes spatiales et matérielles, comment les serviteurs non haïtiens sont intégrés dans le culte, à l'instar de ceux qui composent la famille spirituelle de manbo M. ?

J'ai abordé la présence des Antillais dans ce temple à partir d'une question centrale : comment les dispositifs sociaux, rituels et discursifs révèlent-ils les frontières ethniques 
et les relations de pouvoir à l'intérieur du culte ? Au cours des échanges avec la fille de manbo M. et de l'observation du déroulement de plusieurs cérémonies dans leur temple, j'ai constaté des procédés d'inclusion des Antillais dans le culte et le maintien discret de frontières ethniques entre serviteurs haïtiens et serviteurs antillais. Manbo R. précisa dans un entretien que sa pratique du vodou fut tardive, partagée qu'elle était entre sa vie sociale, son entourage amical français et les activités religieuses de sa mère qui impliquaient de retourner en Haïti. ${ }^{14}$ Plus tard, elle insista sur son attachement au culte en soulignant son caractère atavique, fondé sur le sang, les racines et la race :

« On dit toujours que les dieux des vaincus sont les diables des vainqueurs [...]. Après avoir fait la traversée, j'aime bien cette image, je n'ai pas fait la traversée en bateau, enchaînée, mais c'est dans mon sang, c'est dans mes racines et à chaque fois que je suis en bas [dans la cave] et qu'il y a une cérémonie, et bien je retrouve cette racine. [...] Comme le disent la majorité des gens qui arrivent ici la première fois, qui sont ... on va dire d'origine africaine, et c'est plus fort encore quand ils vont en Haït, ils disent : "ce n'est pas possible, on m'attendait! Il y a quelque chose, ce n'est pas possible! Comment ça se fait?" ». (Manbo R., 15 mai 2003)

Pour désigner les Antillais, majoritaires par rapport aux Haïtiens, qui fréquentent ce temple, manbo R. utilise l'expression «d'origine africaine ». L'Afrique et l'esclavage permettent d'établir une origine et une filiation communes entre les Haïtiens et les Antillais. L'évocation du « sang », des « racines » et de l' « origine africaine 》 montre que les critères de l'appartenance au vodou peuvent être autres que strictement religieux et sont autant de « ressources pouvant être mobilisées pour entretenir ou créer le mythe de l'origine commune » (Poutignat et Streiff-Fénart 1995 [1999] : 178). Cependant, si cette origine commune postulée permet d'inclure les Antillais dans le vodou, j'ai remarqué une gestion sélective des transes de possession au cours des cérémonies. En effet, alors que certains des Antillais composant la famille spirituelle sont manbo ou oungan, seules manbo R. et sa mère étaient chevauchées par les lwa de façon accomplie, les transes de possession étant menées à leur terme. Dès que les ounsi [initié/e] antillaises manifestaient un début de transe incontrôlée, elles étaient conduites à l'extérieur du temple, par un escalier à l'accès limité. D'après ce que j'ai pu observer dans des cérémonies réalisées à Badjo et à Soukri, près des Gonaïves en Haïti, les personnes qui sont l'objet de ce type de transe, parfois provoquée par un lwa bosal -qui n'a pas été fixé dans la tête de l'initié restent dans l'enceinte du temple.

Comment saisir l'attrait des Antillais pour le vodou alors qu'en Guadeloupe une partie de la population est très hostile aux migrants haïtiens (Brodwin, 2003; Ducosson, 2007; Giraud 2002; Hurbon, 1983; Reno, 2008) ? Les Haïtiens seraient-ils plus légitimes et plus compétents que les Antillais pour servir les esprits et être possédés par eux? Tous les oungan et manbo rencontrés en Ile-de-France m'ont affirmé que les Antillais étaient majoritaires, aussi bien parmi leurs consultants que parmi les pitit fey qu'ils ont initié. Plusieurs éléments permettent de comprendre cet attrait de certains Antillais pour le vodou. D'une part, la coexistence de l'origine africaine, de la maji (Adouane, 2000-2001 : 83) et des pratiques thérapeutiques (Benoît, 2000 : 189), est centrale dans cette dynamique d'attraction et de répulsion que suscite le culte. D'autre part, les circonstances 
historiques et le passé colonial sont à considérer pour comprendre la complexité des relations entre les Haïtiens et les Antillais. ${ }^{15}$ Enfin, la transe de possession n'est ni la propriété exclusive ni la qualité naturelle d'un groupe d'appartenance, qu'il soit religieux, national ou autre. Comme j'ai pu le constater en Haïti, les transes de possession peuvent être imitées et pratiquées dès le plus jeune âge et elles nécessitent de travailler des compétences mentales et physiques ce qui, d'un point de vue religieux, est l'un des objectifs du processus initiatique.

Dès lors, le maintien discret de frontières ethniques entre Haïtiens et Antillais quant à la gestion de la transe dans ce temple révèle l'existence de relations de pouvoir au sein du culte. Ces relations sont également fonction de la détention de la konesans [connaissance] qui fonde l'autorité et le charisme des oungan et des manbo :

Étant donné que le principe même de consécration réside dans le fait que l'idéologie et la pratique religieuse remplissent une fonction de connaissance-méconnaissance, les spécialistes religieux doivent nécessairement se cacher et cacher que leurs luttes ont pour enjeu des intérêts politiques, parce que l'efficacité symbolique dont ils peuvent disposer dans ces luttes en dépend et qu'ils ont donc un intérêt politique à cacher et à se cacher leurs intérêts politiques. (Bourdieu, $1971:$ :316-317)

Alors que le vodou est l'un des motifs de la stigmatisation des migrants haïtiens dans la Caraïbe, le processus migratoire en France donne lieu à un renversement des rapports de domination entre Antillais et Haïtiens. Du fait de leur nationalité et du rôle du vodou dans leur histoire nationale, ces derniers sont appréhendés, et ils se présentent ainsi, comme les détenteurs légitimes d'un savoir religieux et magique réputé pour sa puissance. La gestion sélective de la transe dans ce temple signifierait que l'appartenance haïtienne et les liens du sang prévalent sur une origine commune, en l'occurrence africaine, et sur la parenté religieuse qui lient manbo M. à sa famille spirituelle.

La présence d'Antillais dans le culte en Ile-de-France génère également certaines adaptations rituelles. Celles-ci s'inscrivent dans les pratiques circulatoires des acteurs du vodou entre Haïti et les différents espaces de la diaspora. Dans le contexte de la transnationalisation des communautés religieuses (Capone, 2004; Frigerio, 2004; Mary, 2000; Vertovec, 2000), le culte devient un espace à l'intérieur duquel des sociabilités - les conflits, les rumeurs et la compétition - sont autant d'instruments mobilisés dans les quêtes de prestige et les rapports de pouvoir mettant en jeu la légitimité des acteurs religieux.

\section{Jalousies, rumeurs et tripotay : circulations dans un espace transnational de moralité}

Dans le vodou haïtien, ces sociabilités balisent les pratiques légitimes du culte assorties de prescriptions rituelles et d'un rapport normatif au territoire haïtien. À cet ancrage local, se superposent les pratiques d'un vodou déterritorialisé et adapté à un public non haïtien. Ces transformations sont sanctionnées par la circulation des rumeurs qui révèle l'existence d'un espace transnational de moralité. Ce dernier est délimité par des normes sociales et religieuses indiquant ce qu'il est acceptable, ou non, de rendre visible et ce qu'il est possible, ou non, de faire. 
Les relations de pouvoir et les fondements de la légitimité des ougan et des manbo relèvent d'une gestion des ressources humaines. Celle-ci concerne les rapports entre les acteurs religieux et mobilise les catégorisations du vodou en fonction des différences rituelles régionales haïtiennes et de la finalité sous-tendue par les diverses pratiques du culte. Les catégories à l'œuvre dans le vodou s'inscrivent tout d'abord dans le territoire haïtien, tel que le vodou fran qui désigne le culte pratiqué dans les campagnes, lieu originel de formation du culte. Ce vodou fran fut invoqué par un tambourineur francohaïtien pour critiquer le culte exercé par manbo M. dans son temple francilien : « le vodou haïtien d'ici n'a rien à voir avec le vodou fran tel qu'il se pratique en Haïti, il n'est haïtien que parce qu'il et pratiqué par des Haïtiens qui escroquent les Antillais, c'est d'ailleurs pour ça qu'il n'y a pas d'Haïtiens dans leurs cérémonies ». ${ }^{16}$ J'appris par la suite que ce tambourineur, formé en Haïti, avait eu quelques difficultés pour se faire rétribuer ses prestations musicales dans le temple de manbo $\mathrm{M}$.

D'après un jeune serviteur originaire de Pont-Sondé et étudiant à Paris, plusieurs éléments permettent de différencier les ougan makout, ceux de l'Artibonite, sa région natale, des oungan asogwe, initiés eux dans le sud d'Haïti et dans la région métropolitaine de Port-au-Prince :

" Le ougan makout c'est un oungan qui est en dehors, c'est à dire l'Artibonite. Nousmêmes, on est initié, on fait un sevis tet mais on ne prend pas l'ason. [... ] Le oungan makout n'a pas le tcha-tcha du oungan asogwe. [...] Un ougan asogwe est obligé d'aller apprendre auprès d'un ougan pendant 41 jours avec Papa Loko nan bwa. Nous, on a pas besoin d'être initié pour appeler un lwa ou pour diriger un service [...]. Eux, ils sont plus folkloriques que nous autres. Chez eux, tous les enfants peuvent être oungan, chez nous, non. [...] Chez nous, il faut être choisi par les mystères pour être oungan et ce choix peut être fait dès l'enfance. Chez eux, on peut se lever le matin et dire : "tiens, je vais me faire asogwe" ». (Saint-Denis, 28 novembre 2007)

À cette démarche volontariste qui différencie les ougan asogwe des oungan makout et qui s'inscrit dans des particularités rituelles régionales, s'ajoutent des catégories permettant de différencier les finalités du vodou. Dans les rapports entre le culte et la magie, au moins deux modèles coexistent dans une relation d'interdépendance délimitant les prérogatives et les champs d'action de chacun de ces modèles. Cette relation dialectique a été soulignée par Karen Richman à travers sa définition des termes ginen [Guinée] et maji [magie] qui désignent « deux systèmes moraux ou façons d'être au monde » (Richman. 2005 : 17). ${ }^{17}$ Cette relation entre des réalités et des finalités antagonistes, ginen versus maji, distingue également les oungan ginen, qui font le bien, des oungan djab, qui font le mal. Elle est articulée à d'autres formes de différenciation relatives au territoire. À l'inscription dans le territoire rural se superposerait une relation légitimante à l'origine africaine, ginen fran et vodou fran, et des différences régionales : liturgie, modalités de l'initiation, identité des lwa, danses, etc. Le vodou pratiqué en dehors d'Haïti s'inscrit dans ces clivages, tout en en produisant d'autres. De plus, du fait de sa déterritorialisation, il accuse une perte de légitimité à l'instar des autres cultes afro-américains relevant des « diasporas religieuses secondaires 》 (Frigerio, $2004:$ 42). 
Les critiques formulées par tel acteur religieux à l'égard d'un autre peuvent s'appuyer sur ces clivages mais ils révèlent aussi la compétition qui existe dans le vodou. Ainsi, à la demande de manbo S. venant d'arriver d'Haïti, je la conduisis dans le temple de manbo M. à l'occasion d'un service en l'honneur des Gédé. Cependant, alors que l'usage veut que oungan et manbo se saluent par un court rituel appelé dogwe qui marque le respect mutuel et la reconnaissance du statut de chacun, manbo M. ne salua pas cette manbo de passage à Paris. Après la cérémonie, sur le chemin du retour, cette dernière critiqua vivement ce qu'elle avait vu et entendu : « Tout ça c'est pas vrai, c'est pas naturel, c'est du show biz! Il n'y a pas de regleman et les initiés ne disent que des betiz [paroles grivoises] » (Paris, 31 octobre 2004). ${ }^{18}$ Mais ces propos peu amènes résultent également du refus de manbo $\mathrm{M}$. de saluer sa compatriote. D'après oungan R. qui fréquenta son temple, manbo M. ne salue jamais les oungan et les manbo qu'elle n'a pas initié. En effet, le dogwe marquant l'égalité des relations de pouvoir entre ceux qui se saluent ainsi, sa non réalisation manifesterait la volonté de ne pas reconnaître l'autorité de l'autre et serait liée, dans ce cas, à la volonté de neutraliser toute concurrence potentielle. En effet, et ce risque est réel dans le vodou, manbo M. pouvait craindre que manbo S. n'en profite pour lui «subtiliser » certains de ses initiés antillais. Cette gestion des ressources humaines, avec le risque de circulation ou de fuite des initiés, s'inscrit dans les relations de pouvoir consécutives au processus initiatique : le rapport à l'autorité est au cœur de la filiation entre un/e papa/manman kanzo [père/mère d'initiation] et son/ses pitit fey. Cette filiation est centrale dans la transmission et dans l'apprentissage du vodou. Cette gestion des ressources humaines vise donc à minimiser les risques quant aux atteintes contre le prestige des chefs de culte.

L'un des moyens d'affirmer sa légitimité par rapport aux autres acteurs religieux (Capone, 1999 : 147), tout en préservant sa propre réputation, réside dans la circulation des rumeurs et des tripotay [commérages]. Ces derniers constituent des tentatives de contrôle de transgressions, supposées ou réelles, ayant lieu dans les pratiques du culte en Ile-de-France. Indexés à des normes sociales et religieuses, ces rumeurs et commérages forment, du fait de leur circulation d'un bord à l'autre de l'Atlantique, ce que j'appelle un espace transnational de moralité. « Le commérage possède une traçabilité dont ne dispose pas la rumeur » (Bougerol, 2010 : 133), cette dernière consistant à « livrer un récit ou émettre une opinion à l'adresse d'un auditoire en se plaçant derrière un paravent impersonnel et anonyme ("il se raconte que ...") » (Aldrin, 2003 : 127).

J'exposerai ici un cas de rumeur concernant une accusation de sacrifices humains et un exemple de commérage au sujet d'un mariage mystique, ces deux événements ayant eu lieu dans le temple de manbo M. Retracer la genèse, le contenu et le fonctionnement de cette rumeur et de ce commérage permet d'articuler la pérennité de normes sociales haïtiennes dans la migration à l'existence de prescriptions rituelles dans le vodou.

Le fils de manbo M. me fit part d'une accusation de sacrifices humains dont ils avaient été victimes. Selon lui, il n'y a pas d'Haïtiens dans le temple, si ce n'est les membres de la famille, car la réussite de sa mère susciterait trop de jalousies. Les tripotay qui visaient sa mère lui reprochaient de ne pratiquer le vodou que pour l'argent. Son fils soupçonnait alors que leur téléphone soit sur écoute car certaines informations sortaient du temple alors qu'elles ne le devraient pas et cela ne pouvait venir d'un membre de leur famille spirituelle. La circulation des tripotay en dehors du temple permettait à la police 
d'en suivre les activités. Un jour, plusieurs policiers arrivèrent pour arrêter les membres de la famille de manbo M., à la suite d'une dénonciation anonyme les accusant de sacrifier des enfants dont les restes auraient été enterrés dans le sol de la cave. Après enquête, il s'avéra qu'il s'agissait d'une calomnie, mais les policiers continuèrent à suivre les activités qui se déroulaient dans le temple, certains d'entre eux venant consulter manbo $\mathrm{M}$. pour leur propre compte étant impressionnés, selon son fils, par son pouvoir. L'accusation de sacrifices humains et sa diffusion par la délation, qui illustre ici l'aboutissement de la rumeur sanctionnant la réussite économique de cette manbo, doit être située à partir du contexte social haïtien et caribéen. Ce dernier serait dominé par une quête égalitariste pénalisant par les médisances et la jalousie l'individu qui se distingue d'une manière ou d'une autre (Barthélemy, 1991 [1989] : 28-34 ; Price, 1964 : 100). ${ }^{19}$ En Haïti, « toute richesse est censée être acquise par la sorcellerie » (Hurbon, 1979 : 135) et cet enrichissement consécutif à un acte de sorcellerie implique souvent le sacrifice d'un enfant (Laferrière, 2001 [1991] : 92-93 ; Richman, 2005 : 18). Dans le cas de manbo M., l'accusation de sacrifices humains avait pour objectif de nuire à sa réputation et d'attribuer une origine à son enrichissement qui, selon les interlocuteurs, est interprété de deux façons, la réussite versus l'escroquerie, comme le dénonçait le tambourineur franco-haïtien cité plus haut.

Ulf Hannerz souligne dans son analyse des ragots et des réseaux dans un ghetto noir américain que « le commérage est d'abord l'affaire d'individus et, en second lieu, éventuellement, l'instrument d'un système de normes » (Hannerz, 1983 [1980] : 238). Cette analyse permet de comprendre la diffusion du commérage concernant les protagonistes d'un mariage mystique dans le temple de manbo M. Ce rituel consiste à se placer sous la protection d'un lwa, l'initiative pouvant être prise aussi bien par ce dernier que par le serviteur. Karen McCarthy Brown relate les circonstances du mariage mystique entre Mama Lola et le lwa féminin Erzuli Dantor :

Dans ces rituels, les protagonistes se promettent loyauté, service et toujours fidélité sexuelle pendant une nuit de la semaine (ne pas dormir avec un humain pendant cette nuit et attendre de recevoir l'esprit dans leurs rêves), en échange de quoi l'esprit accorde attention et protection. Et encore, quand le ragot a cours, les gens de la communauté d'Alourdes [Mama Lola] admettent que certains de ces mariages se font avec une femme. (Brown, 2001 [1991] : 228).

Le commérage concerne ici l'identité sexuelle des époux et s'inscrit dans cette articulation entre le religieux, le genre et la sexualité décrite par Elizabeth McAlister (2004). Une rapide description du mariage mystique auquel j'ai assisté s'impose. Au cours d'un service marquant la sortie des initiés à l'issue de leur kanzo [initiation], l'un des fils de manbo M. manifesta les signes d'une transe par Ogou et fut paré des attributs matériels de ce dernier : sabre, cigare, foulards et bouteille pailletée rouge. Après les chants déclinant les différents lwa de l'escorte d'Ogou, ce dernier sacrifia une poule rousse et fut conduit avec une initiée et son bébé à l'extérieur du temple. À leur retour, ils furent installés sur deux fauteuils et un homme de l'assistance, après avoir décliné l'identité des protagonistes, les invita à échanger leur consentement mutuel. Ensuite, Ogou et la mère du bébé signèrent l'acte de mariage à l'aide d'une bougie rouge, le lwa passa une bague 
surmontée d'une pierre rouge au doigt de la mère et il donna à son bébé une tétine rouge. Il s'avéra que le rituel consacrait en fait l'union entre Ogou et ce bébé âgé de quelques mois.

Pourquoi les commérages s'emparèrent-ils de cet événement? S'il arrive qu'un enfant soit placé sous la protection d'un lwa, le mariage entre un lwa et un bébé semblait cependant contraire aux prescriptions rituelles coutumières. Il s'agissait de faire connaître cette transgression par rapport aux usages : ce bébé, de sexe féminin, ne pouvait assurer toutes ses obligations envers son époux, pour lequel une nuit de la semaine devait lui être consacrée. Cette transgression fut diffusée bien au-delà du temple de manbo M. car oungan R., résidant alors à Brooklyn, en fut informé par une de ses connaissances rencontrées à Miami. À l'occasion d'un service en l'honneur des Gédé réalisé dans le temple de ce oungan, j'ai interrogé l'un des serviteurs pour savoir si un mariage entre un bébé et un lwa était possible, ce à quoi il me répondit, sur le ton de la raillerie : « tu parles de ce qui s'est passé dans le temple de manbo M. ? ». Cet événement qui avait eu lieu en Ile-deFrance alimenta par la suite les conversations entre certaines des personnes qui assistèrent à ce service dans ce temple de Brooklyn.

La circulation des commentaires sur cet événement par-delà l'Atlantique souligne tout d'abord le rôle des moyens de communication, à commencer par le téléphone, dans la diffusion presque instantanée des informations. Cette circulation est constitutive de milieux sociaux, circonscrits et isolés à l'image de ce temple en région parisienne, qui participent d'un maillage en réseaux grâce aux acteurs qui y sont investis. De plus, la circulation de la rumeur et du commérage, en tant qu'usage social de la norme - de ce qu'il convient de dire et de ce qu'il convient de faire - balise un espace transnational de moralité délimitant les pratiques légitimes du culte. Rumeur et commérage montrent également que, s'ils se déploient dans le milieu du vodou en diaspora, ils se réfèrent à des pratiques sociales spécifiques au contexte rural haïtien. Plusieurs chercheurs, à l'instar de Jonathan Friedman, ont critiqué le recours excessif au modèle du transnationalisme dans les discours anthropologiques, car ils relèveraient d' « un programme élitiste dont la vision d'en haut, s'appuie [...] sur l'expérience du vol aérien ». Mais la circulation des rumeurs et commérages souligne au contraire que les serviteurs du vodou, et les migrants, participent pleinement aux réseaux transnationaux (Capone, 2004 : 11). Ces procédés discursifs de contrôle de transgressions par rapport aux usages rituels permettent certes de délégitimer tel concurrent. Mais ils sont également exemplaires de ces tentatives de maintenir, et parfois de construire, une orthodoxie face à la plasticité et à la dissémination des modèles rituels portés par des acteurs religieux " mondialisés ».

\section{Conclusion}

Entre la fin des années 1930 et le début des années 1970, on observe une transformation très nette des finalités du folklore haïtien et des pratiques du vodou. Ils ont constitué le principal objet des études académiques, autant haïtiennes qu'étrangères, dans un contexte où le culte faisait l'objet d'une pénalisation juridique, du moins dans les textes. La dissociation entre ce qui relève du folklore et ce qui appartient au religieux a permis de sélectionner, dans le vodou, ce qui pouvait faire l'objet de mises en scène publiques, la transe et le sacrifice en étant a priori exclus. ${ }^{20}$ Les troupes folkloriques haïtiennes 
participèrent à l'affirmation d'une conscience noire sur la scène culturelle haïtienne et internationale et à un objectif économique consistant à satisfaire les attentes exotiques des touristes et du public, haïtien comme étranger. Les archives du Ministère haïtien des Affaires étrangères montrent que les danseurs les plus en vue furent investis d'une mission de communication sur l'image d'Haïti, dynamique qui souligne la force d'une esthétique politique du vodou dans ce dispositif de nationalisation de la culture populaire haïtienne. Cependant, l'amplification des difficultés sociales, économiques et politiques pendant le règne de Jean-Claude Duvalier (1971-1986) a fait des troupes folkloriques un vivier de compétences artistiques mobilisées pour l'exil. Vu du contexte haïtien, ces mises en scène du culte révèlent les rapports de pouvoir sur lesquels s'appuyait l'investissement de membres de l'élite haïtienne dans leur valorisation intéressée et ambiguë du vodou. Les parcours de Mathilda Beauvoir, par laquelle les show vodou sont arrivés en France, et de l'une de ses anciennes danseuses soulignent l'étroite articulation entre ces compétences et un système organisé de filières migratoires. Ils illustrent également les enjeux de ces mises en scène du culte associant étroitement l'art et le religieux. Ces enjeux se posent par rapport au contenu de ces mises en scène, avec la limite entre ce qui est montrable et ce qui doit demeurer caché, et au sujet du double statut des acteurs de ces spectacles, avec la question de leur crédibilité et de leur légitimité en tant qu'artistes et spécialistes religieux. La construction des savoirs sur le vodou, aussi bien dans le passé qu'aujourd'hui, est socialement et politiquement située. Ainsi, enquêter sur le vodou quand on est étranger en tout point à ce culte, implique une posture de recherche à la fois distancée et impliquée. Cette posture participe, en les révélant, des rapports de pouvoir qui sont autant constitutifs au vodou, en tant que religion initiatique dont le pivot est le secret, qu'extérieurs à lui, du fait de sa présence dans une société française globalement hostile à ce culte. De cette présence découle toute une série d'adaptations relationnelles, matérielles et rituelles qui répondent aux contraintes de l'environnement urbain francilien et qui soulignent la plasticité des pratiques du vodou. Dès lors, l'invisibilité et la discrétion s'imposent, aussi bien pour masquer des sacrifices prohibés par la loi que pour cacher des échanges économiques le plus souvent informels. Ce culte peut être appréhendé comme un espace commercial transnational dès lors qu'il donne lieu à des liens d'interdépendance et à des pratiques de solidarité autour d'acteurs religieux investis dans les dynamiques migratoires entre Haïti et l'étranger. La circulation de ces acteurs et leur inscription dans des sociétés postmigratoires, où les individus manifesteraient le besoin d'ancrages particuliers (Schnapper, 1993 : 157), nécessitent des adaptations à un public non haïtien. Dans le contexte migratoire francilien, la présence massive des Antillais confère au vodou une fonction de cohésion, liée à un sentiment d'exil et postulée à partir d'une origine commune africaine. Elle donne également à voir le maintien discret de frontières ethniques, confirmant ainsi le statut des Haïtiens en tant que détenteur légitime d'un capital religieux réputé autant pour sa puissance que pour son caractère authentique. Cette dynamique révèle le renversement des rapports de domination entre Antillais et Haïtiens et elle génère des ajustements rituels et des profits économiques, liés à un élargissement du public du culte. Dès lors, cette réussite et ces transformations sont sanctionnées par des rumeurs et des commérages. Parce que ces derniers se déploient en fonction de normes sociales et de différences rituelles forgées en Haïti, et parce qu'ils circulent d'un bord à l'autre de l'Atlantique, ils aboutissent à la formation d'un espace transnational de 
moralité. Ces divers usages sociaux du vodou en contexte migratoire se fondent sur des hiérarchies sociales et religieuses entre lesquelles des acteurs circulent en jouant des relations de pouvoir. Ces hiérarchies et ces relations confèrent au culte une dimension éminemment politique et transnationale.

\section{Notes}

1. Les analyses proposées dans cet article se fondent sur une ethnographie multi-située des pratiques du vodou entre Haïti, l'Ile-de-France et Brooklyn, menée de 2002 à 2008 dans le cadre d'une thèse d'anthropologie sociale et ethnologie : 2010, Les pratiques migratoires entre Haïti et la France. Des élites d'hier aux diasporas d'aujourd'hui (dir. M.-J. Jolivet), Centre d'Études africaines, École des Hautes Études en Sciences Sociales (563 p.).

2. «Sur la suggestion de plusieurs personnes (officiels et journalistes américains) qui eurent l'occasion d'admirer notre folklore à l'Exposition, une place spéciale nous est réservée dans le programme [... ]. La participation d'Haïti lui vaudrait, selon Monsieur Lever, une propagande touristique d'un million de dollars environ »; « . . . la portée de cette manifestation qui représente pour nous à n'en pas douter, une rare opportunité de combiner la meilleure forme de publicité et l'affirmation de notre culture dans les Amériques, le Gouvernement a pris les dispositions nécessaires pour s'y faire représenter, comme il convient, par la Troupe Folklorique Nationale ». Extraits des lettres du 2 février et du 5 mars 1951 de Joseph L. Dejean (délégué d'Haïti auprès de l'Organisation des États Américains) à Jacques N. Léger (secrétaire d'État aux Relations extérieures). Dossier « Correspondances générales ; Commémoration du centenaire du drapeau haïtien ; Participation de la Troupe folklorique et de Caridad Appolon au Festival des Cerisiers en Fleurs, Washington », (A.AE) A.C./Sig1/1951/97(2), Ministère des Affaires Étrangères, Port-au-Prince.

3. «Vendredi soir dernier, l'Hôtel Diplomat fut le centre d'une soirée unique dans les annales de New York. Jean-Léon Destiné fut le grand artiste de la soirée. [...] En le voyant danser, en l'entendant chanter, nous avons découvert les nouvelles facettes de l'âme haïtienne ... les artistes d'un certain pays font mieux connaître - et plus avantageusement - leur propre nation que tous les bureaux de renseignements et toutes les agences publicitaires » (M. Lacoste, France Journal du 8 juin 1948, cité par Oriol, Viaud et Aubourg 1952 : 87-88). Jean-Léon Destiné fut recruté en 1946 par Katherine Dunham qui dirigeait à New York une école de danse, puis il créa en 1949 sa propre compagnie, la Destiné Afro-haitian Dance Company. En 1960, il devint attaché culturel d'Haïti aux États-Unis.

4. En 30 ans, de 1946 à 1978, le nombre de touristes passa de 10000 (Smith, 2009 : 164) à 300 000 (« Tourist and Excursion Arrivals in Haiti 1951-1978 », ONRTP, Institut haïtien des statistiques, cité par Goldberg, $1981: 175)$.

5. Katherine Dunham (1909-2006) fut étudiante en anthropologie à l'Université de Chicago et, soutenue par Melville Herskovits, elle débuta ses recherches en Haïti en 1935 grâce à une bourse de la Rosenwald Foundation pour étudier les danses des Caraïbes (Capone, 2006 : 92). Elle s'initia au vodou et acquis dans les années 1940 l'habitation Leclerc. À côté de cette habitation, située dans le quartier de Martissant à l'ouest de Port-au-Prince, elle possédait également une résidence où elle produisait des spectacles vodou.

6. Article de la revue Arts du 2 novembre 1951, intitulé « Katherine Dunham et Cicéron ont fait pleurer Charlot » et transmis par l'Ambassade d'Haïti à Paris au Secrétariat des relations extérieures. Lettre du 19 octobre 1951 du Général Lavaud à Jacques Léger. Dossier : 
" Correspondances avec l'Ambassade d'Haïti en France », (A.AE) A.C./Sig.2/1951(14), Ministère des Affaires étrangères, Port-au-Prince.

7. Entretien téléphonique avec Françoise Morin, 10 juin 2009.

8. «Depuis six années consécutives [depuis 1968], je vis en contact permanent avec des groupes de vaudouïsants qui logent dans mon propre jardin. Les choses se passent de la manière suivante : chaque été, Mathilda et moi-même nous nous rendons en Haïti et en revenons accompagnés d'une dizaine de "hounsi" choisi dans divers temples de l'île. [...] Tous ont été confiés à "Graine Promenée" [nom vaillant de Mathilda Beauvoir] par leur "houngan" ou "mambo" d'origine, dont elle prend le relai pour une durée déterminée. D'ailleurs, dans bien des cas, elle a participé elle-même à leur initiation : on profite toujours, en effet, de son passage à Haïti pour faire entrer dans le "guévo" [ou djèvo, chambre de réclusion initiatique] les garçons ou filles susceptibles de venir à Paris, dans un avenir plus ou moins éloigné » (Planson, 1975 [1974] : 191).

9. « D'abord, un trafic d'être humains lucratif qui a débuté dans les années soixante-dix. Il consistait à faire venir des compatriotes à Paris en les faisant passer pour des danseuses professionnelles. Bien que cette activité soit réelle, l'usage qu'en faisait la prêtresse du vaudou était tout à fait contestable. [ . . ] De 1970 à octobre 1998, plus d'une cinquantaine de compatriotes, essentiellement des femmes, sont arrivées à Paris grâce à son réseau ». Maguet Delva, Haiti Caraibe Express, $\mathrm{n}^{\circ}$ 8, 20 nov.-3 déc. $2002: 1$ et 10.

10. « Nous nous en sommes aperçus avec ceux, rares d'ailleurs, qui, leur "contrat" terminé, ont voulu rester en France. À l'exception d'un seul, aucun n'a pu s'y faire une place décente et tous ont perdu ce qui faisait leur force. Éloignés du vaudou, ils n'étaient plus qu'une maind'œuvre sans qualification, et rejoignait très vite ce sous-prolétariat des villes, toujours plus ou moins en situation illégale [ ... . Mais l'expérience a montré qu'il ne fallait jamais les laisser trop longtemps coupés de leurs racines, sous peine de les voir perdre les qualités propres à leur peuple : le courage, la simplicité, la gaieté et un sens artistique exceptionnellement développé » (Planson, 1975 [1974] : 193).

11. La problématique de l'initiation comme voie d'accès à la connaissance et comme mode privilégié d'enquête de l'ethnographie a trouvé dans les religions afro-américaines en général, et dans le vodou en particulier, un terrain de choix. La figure du « chercheur initié » a notamment été incarnée par Karen McCarthy Brown qui relate son initiation dans son ouvrage Mama Lola (Brown, 2001 [1991] : 312-328). L'on se réfèrera également à la réflexion de Stefania Capone qui souligne avec justesse la complexité des enjeux noués autour de l'initiation des chercheurs. Condition indispensable pour pénétrer à l'intérieur du culte étudié, l'initiation peut être mobilisée dans la recherche d'un surplus de légitimité quant au savoir produit : « Cette appartenance donne une autorité toute spéciale à la voix de l'ethnologie : elle entraînerait, ipso facto, la connaissance de la culture étudiée. L'ethnologue devient une sorte d'intellectuel organique du culte (Capone, $1999: 44)$.

12. La sorcellerie serait un "pouvoir supposé que certains hommes peuvent, par l'effet de qualités ou de techniques innées, héritées ou acquises, exercer sur d'autres» et «l'un des langages dans lesquels s'expriment les rapports de force sociaux » (Augé, 1982 : 255). En Haïti, la forte aspiration égalitaire dans une société marquée par les défaillances de l'État fait que « la sorcellerie devient ainsi le régulateur des rapports de crise entre les individus » (Barthélemy, 1991 [1989] : 35). L'accusation de sorcellerie permet de donner un sens et d'attribuer une origine au malheur, à la souffrance et à la maladie (Farmer, 1996 [1992] : 275-372). André 
Corten (2001 : 48) et Laënnec Hurbon (1979 : 133-134) situent le développement des accusations de sorcellerie et le recours à cette dernière dans le délitement général de la société haïtienne. Les serviteurs du vodou différencient ce qui relève du ginen - qui peut être traduit par « tradition »et ce qui relève de la maji, la «magie », qu'elle soit bénéfique ou maléfique (Richman, 2005 : 150-168).

13. Tout comme en Haïti, où le commerce lié au vodou fit l'objet de condamnations juridiques par un décret-loi promulgué en 1935 (Hoffmann, 1990 : 138) - commerce qui continue à être au cœur des accusations de charlatanisme à l'intérieur même du vodou - à Cuba, le mercantilisme au sein de la religión est également réprouvé : «Les plus vieux en religión se plaignent d'ailleurs de ce raz de marée, accusant vaguement "certains" de "vouloir exploiter la religión", de pratiquer mercantilisme et charlatanisme et de divulguer à n'importe qui les secrets rituels » (Argyriadis, $1999: 40$ ).

14. "C'était vraiment ce qu'il y avait de plus difficile à concilier, être la fille de manbo M. et d'être moi-même. J'ai fait mes classes ici, tous mes amis sont européens, c'était très difficile de concilier les deux. Et puis en fin de compte, je me suis rendue compte qu'il n'y avait pas de complications à faire, qu'il n'y avait plus de frontières. [... ] Mais c'est vrai que quand tu es à l'âge de la puberté, tu as les copines qui partent en vacances et toi, tu dois aller en Haïti parce qu'il y a une cérémonie, tu n'arrives pas à comprendre tout de suite, à l'accepter » (Manbo R., 27 novembre 2002).

15. « Il y a en effet toute une ambiguïté de conduite chez l'Haïtien à l'égard du Martiniquais ou du Guadeloupéen. Il se sent à la fois proche de par la situation géographique (les Caraïbes) et le passé historique (l'esclavage) et très éloigné car il le méprise et lui reproche encore d'être un "colonisé" et de ne pas avoir la fierté du peuple haïtien, libre depuis Toussaint Louverture » (Bastide, Morin et Raveau, 1974 : 402).

16. Travaux publics, émission de la radio France Culture, 3 mars 2004.

17. «Guinée (ginen) signifie tradition, interdépendance et autorité morale. Le terme Guinée se réfère au lointain, au lieu mythique "de l'autre côté de l'eau" d'où les ancêtres migrèrent et vers où il retournent à leur mort, et où les lignées d'esprits continuent à vivre. Le terme Guinée s'incarne aussi dans le concept complexe d'héritage (eritaj), qui représente à la fois la terre inaliénable, héritée par parenté directe, celle de leurs ancêtres paysans, et leur héritage spirituel. La magie est la Guinée de l'Autre, sa figure souterraine. La magie est associée au travail salarié, à l'extérieur, à l'individualisme débridé et, donc, à la sorcellerie. Le travail de la magie est supposé être exécuté par un genre d'esprits connu sous le nom de pwen. [...] Les pwen sont fabriqués et vendus par les sorciers » (Richman, $2005: 17-18$ ).

18. Traduit du créole.

19. «Dans ce système, l'égalité entre les participants est le corollaire de l'automatisme de la régulation, car toute inégalité reposant sur une accumulation significative de richesse, par l'un ou l'autre des citoyens, débouche inéluctablement sur un déséquilibre dans le contrôle du pouvoir » (Barthélemy, 1991 [1989] : 28).

20. Des transes de possession pouvaient cependant se produire lors de spectacles folkloriques. C'est ce que rapporte un journaliste qui assista à une soirée mondaine donnée dans le Carlton Hotel à Washington en l'honneur du président haïtien fraîchement élu, Élie Lescot : « La danse du Vaudou, une danse rituelle réservées pour les clairs de lune en Haïti a été une représentation cosmopolite hier avec les diplomates et les membres du Monde officiel regardant les filles nus pieds en calicot [... ]. Ils ont dansé et chanté sur une scène dressée à un bout de la 
longue salle du Carlton, avec de rudes tambours natifs et le doux rythme des mascarons. Ils ont été pris de frénésie quand le chœur devint plus fort et plus insistant en atteignant un crescendo de mouvement quand le "dieu" finalement prit possession de sa victime ». Anonyme, 4-5 mai 1941, « Lescot reçoit avec danseurs du vaudou avant de partir pour occuper la présidence d'Haïti », Le Matin : 2. Je remercie Lewis A. Clormeus pour m'avoir communiqué cet article.

\section{Références}

Adouane S (2000-2001) La pratique du vodou haïtien à Paris. Psychopathologie africaine 31(1) : 69-91.

Aldrin P (2003) Penser la rumeur, une question discutée des sciences sociales. Genèses 50 : $126-141$.

Apter A (2002) On African origins : creolization and connaissance in Haitian Vodou. American Ethnologist 29(2) : 223-260.

Argyriadis K (1999) La religión à La Havane. Actualités des représentations et des pratiques culturelles havanaises. Paris : Éd. des Archives Contemporaines.

Augé M (1982) Génie du paganisme. Paris : Gallimard.

Barthélemy G (1991 [1989]) L'univers rural haitien. Le pays en dehors. Paris : L'Harmattan.

Bastide R, Morin F et Raveau F (1974) Les Haitiens en France. Paris-La Haye : Mouton.

Beauvoir-Dominique R (2005) Fonds documentaire Odette Mennesson-Rigaud. Rapport d'inventaire. Port-au-Prince : AUF / Bibliothèque haïtienne des Pères du Saint-Esprit.

Béchacq D (2004) Commerce, pouvoir et compétences dans le vodou haïtien. Cahiers des Anneaux de la Mémoire 7 : 41-69.

Béchacq D (2006) Les frontières du vodou haïtien. In : Bonacci G, Béchacq D, BerloquinChassany P et Rey N (dir.) La Révolution haitienne au-delà de ses frontières. Paris : Karthala, 253-271.

Béchacq D (2008) La construction d'un vodou haïtien savant. Courants de pensée, réseaux d'acteurs et productions littéraires. Tabou $5: 27-69$.

Benoît C (2000) Corps, jardins, mémoires. Anthropologie du corps et de l'espace à la Guadeloupe. Paris : CNRS/MSH.

Bougerol C (2010) Une rumeur à la Guadeloupe. De certaines pratiques supposées des Haïtiens. Terrain 54 : 130-139.

Bourdieu P (1971) Genèse et structure du champ religieux. Revue française de sociologie 12(3) : 295-334.

Brodwin P (2003) Marginality and subjectivity in the Haitian diaspora. Anthropological Quartely 76(3) : 383-410.

Brown KM (1998 [1995]) Serving the spirits. The ritual economy of Haitian Vodou. In : Cosentino DJ (ed.) Sacred Arts of Haitian Vodou. Los Angeles, CA : UCLA Fowler Museum of Cultural History, 205-223.

Brown KM (2001 [1991]) Mama Lola. A Vodou Priestess in Brooklyn. Berkeley : University of California Press.

Capone S (1999) La quête de l'Afrique dans le candomblé. Pourvoir et tradition au Brésil. Paris : Karthala.

Capone S (2004) À propos des notions de globalisation et de transnationalisation. Civilisations 51(1-2) : 9-22. 
Capone S (2006) Les Yoruba du Nouveau Monde. Religion, ethnicité et nationalisme noir aux États-Unis. Paris : Karthala.

Charlier-Doucet R (2005) Anthropologie, politique et engagement social : l'expérience du Bureau d'ethnologie d'Haïti. Gradhiva (n.s.) 1 : 109-124.

Corten A (2001) Misère, religion et politique en Haïti. Paris : Karthala.

Cosentino DJ (1993)Vodou Vatican : A prolegomenon for understanding authority in a synthetic religion. Caribbean Quartely 39(3/4) : 100-107.

Cosentino DJ (1998 [1995]) Imagine Heaven. In : Cosentino DJ (ed.) Sacred Arts of Haitian Vodou. Los Angeles, CA : UCLA Fowler Museum of Cultural History, 25-55.

Ducosson DJ (2007) Un nationalisme de parade en Guadeloupe. Esprit 2 (332) : 98-104.

Fabre M (1977) La Revue Indigène et le mouvement nouveau noir. Revue de Littérature Comparée 51(1) : 30-39.

Farmer P (1996 [1992]) Sida en Haiti. La victime accusée (Trad. C. Hewlett). Paris : Karthala.

Frigerio A (2004) Re-Africanization in secondary religious diaspora : Constructiong a world religion. Civilisations 51(1-2) : 39-60.

Gaillard R (1993) L'indigénisme haïtien et ses avatars. Conjonction 197 : 9-26.

Giraud M (2002) Racisme colonial, ethnicité et citoyenneté. Les leçons des expériences migratoires antillaises et guyanaises. Hommes \& Migrations $1237: 40-53$.

Goldberg A (1981) Commercial folklore and Voodoo in Haiti : International tourism and the sale of culture. PhD dissertation, Departement of Anthropology, Indiana University.

Hannerz U (1983 [1980]) Explorer la ville. Éléments d'anthropologie urbaine (Trad. I. Joseph). Paris : Les Éditions de Minuit.

Heusch (de) L (1989) Kongo in Haiti : A new approach to religious syncretism. Man 24(2) : 290-303.

Hoffmann L-F (1990) Haiti. Couleurs, croyances, créole. Montréal/Port-au-Prince : CIDIHCA/ Deschamps.

Hurbon L (1979) Culture et dictature en Haiti. L'imaginaire sous contrôle. Paris : L'Harmattan/CNRS.

Hurbon L (1983) Racisme et sous-produit du racisme : immigrés haïtiens et dominicains en Guadeloupe. Les Temps Modernes 441-442 : 1988-2003.

Hurbon L (1987) Comprendre Haiti. Essai sur l'État, la nation, la culture. Port-au-Prince/Paris : Deschamps/Karthala.

Laferrière D (2001 [1991]) L'odeur du café. Paris : Le Serpent à Plumes.

Largey M (2006) Vodou Nation. Haitian Art, Music and Cultural Nationalism. Chicago/London : University of Chicago Press.

Magloire G et Yelvington KA (2005) Haiti and the anthropological imagination. Gradhiva (n.s.) 1 : 127-152.

Mary A (2000) L'anthropologie au risque des religions mondiales. Anthropologie et Sociétés 24(1) : 117-135.

McAlister E (2004) Amour, sexe et genre incarnés : les esprits du vaudou haïtien. Africultures 58 : $111-130$.

Métraux A (1978) Itinéraires 1 (1935-1953). Carnets de notes et journaux de voyage. Paris : Payot. Métraux A (1995 [1958]) Le vaudou haitien. Paris : Gallimard.

Mintz S et Trouillot M-R (1998 [1995]) The social history of haitian vodou. In : Cosentino DJ (ed.) Sacred Arts of Haitian Vodou. Los Angeles, CA : UCLA Fowler Museum of Cultural History, 123-147. 
Nicholls D (1996 [1979]) From Dessalines to Duvalier : Race, Colour and National Independance in Haiti. New Brunswick, NJ : Rutgers University Press.

Oriol J, Viaud L et Aubourg M (1952) Le mouvement folklorique en Haiti. Port-au-Prince : Imprimerie de l'État.

Peslin-Ursu D (2009) Le Théatre des Nations : une aventure théatrale à redécouvrir. Paris : L'Harmattan.

Pierre LN (2008) Les élites haïtiennes : composition et stratégies de pouvoir. In : Lerat C (dir.) Élites et intelligentsias dans le monde caraibe. Paris : L'Harmattan, 59-82.

Planson C (1975 [1974]) Vaudou, un initié parle. Paris : Éd. lu J'ai.

Poutignat P et, Streiff-Fénart J (1995 [1999]) Théories de l'ethnicité. Paris : Presses Universitaires de France.

Price R (1964) Magie et pêche à la Martinique. L'Homme 4(2) : 84-113.

Price-Mars J (2009 [1928]) Ainsi parla l'oncle suivi de Revisiter l'Oncle. Montréal : Mémoire d'encrier.

Ramsey K (2005) Prohibition, persecution, performance : anthropology and the penalization of vodou in mid-20th century. Gradhiva (n.s.) $1: 165-179$.

Reno F (2008) L'immigrant haïtien entre persécution et xénéphobie. Hommes \& Migrations 1274 : $131-142$.

Richman K (2005) Vodou and Migration. Gainesville : University Press of California.

Richman K (2008) Innocent imitations ? Authenticity and mimesis in Haitian Vodou art, tourism, and anthropology. Ethnohistory 55(2) : 203-227.

Schnapper D (1993) Le sens de 1'ethnico religieux. Archives de Sciences sociales des Religions 81(1) : 149-163.

Smith MJ (2009) Red and Black in Haiti : Radicalism, Conflict and Political Change, 1934-1957. Chapel Hill : Univesity of North Carolina Press.

Tippenhauer Y (2008) Les intellectuels haïtiens et le vodou : Fascination et ambiguïté de l'indigénisme. Tabou $5: 87-104$.

Vertovec S (2000) Religion and diaspora. Communication présentée au colloque Nouveaux paysages de la religion en Occident, Oxford University : 27-29 sept.

Wilcken L (1998) The changing hats of Haitian staged folklore in New York. In : Allen R, Wilcken L (ed.) Island Sounds in the Global City : Carribean Popular Music and Identity in New York. New York : Folklore Institute for Studies in American Music, Brooklyn College, 162-183.

Yarborough LW (1959) Ballets d'Haiti. Bamboche Créole, 20th Anniversary. Port-au-Prince/ Frankfurt : Panorama/Bronners Druckerie. 